\title{
Formazione delle scritture alfabetiche in Italia centrale. Riflessioni sul caso dell'etrusco e alfabeti connessi.
}

\section{The making of alphabetic writing systems in Central Italy. Some remarks about Etruscan and related alphabets.}

\author{
Enrico Benelli \\ CNR-ISMed \\ enrico.benelli@cnr.it
}

Riassunto: Il processo di formazione dell'alfabeto etrusco segue principi molto diversi rispetto a processi analoghi che hanno portato alla nascita delle altre scritture alfabetiche di area mediterranea. La ricerca passata ha spesso mancato di cogliere questa anomalia, o ha tentato di spiegarla attraverso modelli teorici non sempre soddisfacenti. Partendo dalla constatazione che le città etrusco-meridionali, al momento della formazione della scrittura alfabetica, comprendevano componenti alloglotte, evidenti soprattutto ai livelli sociali più alti, e introducendo confronti con situazioni analoghe riscontrabili in vari sistemi scrittori del mondo, l'articolo propone di spiegare il singolare processo formativo dell'alfabeto etrusco come il risultato di un tentativo di creare una scrittura che potesse servire a rendere più lingue diverse.

Parole chiave: Etruschi. Alfabeto etrusco. Scritture dell'Italia preromana. Storia sociale etrusca. Multilinguismo nell'antichità.

Abstract: Etruscan alphabet was created following a path that was consistently different from what happened in similar instances in the ancient Mediterranean. Past research has often missed these peculiarities; the rare attempts to explain them have usually involved the elaboration of not always satisfying theoretical models. The paper aims to explain the circumstances surrounding the creation of the Etruscan alphabet by putting it in the proper social and historical context. When the Etruscan alphabet first appeared, Southern Etruscan cities where multilingual communities, including their upper classes. Parallels with writing systems employed elsewhere seem to suggest that the peculiarities of the Etruscan alphabet in its initial stages can be best explained as an attempt to create a writing system which could be used for all these languages at the same time.

Keywords: Etruscans. Etruscan alphabet. Writing in pre-Roman Italy. Etruscan social history. Ancient Multilingualism.

Recepción: 23.01.2020 | Aceptación: 13.03.2020 
0. Le ragioni storico-sociali sottese allorigine della scrittura (epigrafica) etrusca sono state oggetto di numerosi saggi che, nell'ultimo ventennio, hanno raggiunto una sostanziale unanimità di vedute, tanto che non è necessario ritornarvi ulteriormente. Che l'epigrafia, in Etruria, debba le proprie prime manifestazioni a esigenze rappresentative squisitamente aristocratiche è fatto che può considerarsi ormai acquisito alla ricerca, e che difficilmente potrà essere smentito fin tanto che non subentrino nuove scoperte di entità tale da alterare profondamente il quadro documentario attuale. ${ }^{1}$

1. Meno studiato, almeno in tempi recenti, è invece l'aspetto tecnico della forma che prese l'alfabeto etrusco in rapporto al suo prototipo greco, sostanzialmente di matrice euboica. Il dibattito è tuttora centrato su un magistrale contributo del 1990 di Aldo Prosdocimi, posto a commento dell'accurato repertorio degli alfabetari etruschi ad opera di Maristella Pandolfini, ${ }^{2}$ che le successive scoperte hanno certamente arricchito ma non sostanzialmente modificato nel suo aspetto generale. ${ }^{3}$

Tralasciando tutti i punti non controversi, per i quali esiste letteratura abbondante e sostanzialmente concorde, i problemi connessi con la formazione dell'alfabeto etrusco vertono su tre aspetti fondamentali, apparentemente contraddittori:

(1) la conservazione costante negli alfabetari, per circa un secolo (fig. 1), dei segni "morti" $\beta, \delta, \xi /$ samekh, ;

(2) la presenza di due grafemi per $\xi$, quello degli alfabeti greci di tipo "azzurro", nella posizione alfabetica del samekh fenicio, e quello a croce nel comparto terminale dei segni complementari, tipico degli alfabeti "rossi", il cui valore è asseverato sia dal significato di $\chi$ attribuito al segno a tridente (coerentemente con il carattere "rosso" dell'alfabeto euboico che funse da modello a quello etrusco), sia dalla sua effimera rivitalizzazione come grafema di sibilante (e dalla sua persistenza in latino, come $\langle\mathrm{x}\rangle$, con l'originario valore greco "rosso");

1 Per brevità mi limito a rimandare a Benelli c. s. a, che contiene la principale letteratura di riferimento.

2 Pandolfini e Prosdocimi 1990.

3 Lunica rettifica fondamentale al corpus del 1990 è l'acquisizione di una datazione più corretta per la "Tomba dell'Alfabeto" di Monteriggioni (Bartoloni 1997), che peraltro è andata a rafforzare ulteriormente il modello evolutivo degli alfabetari proposto dagli Autori. 
(3) la presenza, anch'essa fortemente contraddittoria a paragone di qualunque modello greco, di due grafemi di sibilante, sigma e san, che nell'uso greco sono sempre alternativi (in greco la forma della sibilante è condizionata da quella di iota, a causa della potenziale omografia: normalmente san concorre con iota a tre tratti, più vicina al modello fenicio, laddove sigma comporta iota lineare; più originale la soluzione documentata dalla sola oinochoe del Dipylon di reciproca inversione di ductus).

SERIE ALFABETICHE DOCUMENTATE DAGLI ALFABETARI ETRUSCHI

\begin{tabular}{|c|c|c|c|c|c|c|c|c|c|c|c|}
\hline \multicolumn{2}{|c|}{ VII secolo } & \multicolumn{4}{|c|}{ VI-V secolo } & \multicolumn{6}{|c|}{ IV-II secolo } \\
\hline & & \multicolumn{2}{|c|}{ sud } & \multicolumn{2}{|c|}{ nord } & \multicolumn{2}{|c|}{ sud } & \multicolumn{2}{|c|}{ nord (IV-III sec.) } & \multicolumn{2}{|c|}{ nord (III-II se } \\
\hline$A$ & a & $A$ & a & A & a & $A$ & a & $A A$ & a & $A A$ & $\mathrm{a}$ \\
\hline$g$ & $\mathrm{~b}$ & כ & c & $\exists$ & e & 3 & c & 3 & e & $\exists$ & $\mathrm{e}$ \\
\hline ) & c & $\exists$ & $\mathrm{e}$ & $\exists$ & $\mathrm{v}$ & $\exists$ & e & $\uparrow$ & $\mathrm{v}$ & 7 & $\mathrm{v}$ \\
\hline$n$ & d & $\exists$ & $\mathrm{v}$ & I & $\mathrm{z}$ & 7 & v & y & $\mathrm{z}$ & $\neq$ & $\mathrm{z}$ \\
\hline$\nexists$ & $\mathrm{e}$ & $I$ & z & 日 & $\mathrm{h}$ & $k$ & z & $\theta$ & $\mathrm{h}$ & 日 & $\mathrm{h}$ \\
\hline 7 & $\mathrm{v}$ & 日 & $\mathrm{h}$ & $\odot$ & $\theta$ & 日 & $\mathrm{h}$ & $\diamond$ & $\theta$ & 0 & $\theta$ \\
\hline I & z & $\odot$ & $\theta$ & 1 & $\mathrm{i}$ & 0 & $\theta$ & I & i & 1 & $\mathrm{i}$ \\
\hline 日 & $\mathrm{h}$ & 1 & $\mathrm{i}$ & y & $\mathrm{k}$ & 1 & $\mathrm{i}$ & $x$ & $\mathrm{k}$ & J & c \\
\hline$\otimes$ & $\theta$ & $x$ & $\mathrm{k}$ & $\checkmark$ & 1 & $\checkmark$ & 1 & J & 1 & $\checkmark$ & 1 \\
\hline 1 & $\mathrm{i}$ & $\checkmark$ & 1 & $m$ & $\mathrm{~m}$ & $m$ & $\mathrm{~m}$ & $M$ & $\mathrm{~m}$ & $H$ & $\mathrm{~m}$ \\
\hline x & $\mathrm{k}$ & $m$ & $\mathrm{~m}$ & 4 & $\mathrm{n}$ & $n$ & $\mathrm{n}$ & $n$ & $\mathrm{n}$ & $\mathrm{H}$ & $\mathrm{n}$ \\
\hline J & 1 & $y$ & $\mathrm{n}$ & 1 & $\mathrm{p}$ & 1 & $\mathrm{p}$ & 1 & $\mathrm{p}$ & 1 & $\mathrm{p}$ \\
\hline$m$ & $\mathrm{~m}$ & 1 & $\mathrm{p}$ & $M$ & ś & $M$ & ś & $M$ & ś & $M$ & ś \\
\hline 4 & $\mathrm{n}$ & $M$ & $\dot{s}$ & 4 & $\mathrm{r}$ & 0 & $\mathrm{r}$ & 0 & $\mathrm{r}$ & 9 & $\mathrm{r}$ \\
\hline 田 & $\xi$ & $\varphi$ & $\mathrm{q}$ & $s$ & s & 2 & s & 2 & $\mathrm{~s}$ & 2 & $\mathrm{~s}$ \\
\hline 0 & 0 & 4 & r & $T$ & $\mathrm{t}$ & $r$ & $\mathrm{t}$ & y & $\mathrm{t}$ & $T$ & $\mathrm{t}$ \\
\hline$\eta$ & $\mathrm{p}$ & $s$ & $\mathrm{~s}$ & $y$ & u & V & u & $V$ & u & $v$ & $\mathrm{u}$ \\
\hline$M$ & śs & $T$ & $\mathrm{t}$ & $\phi$ & $\varphi$ & $\Phi$ & $\varphi$ & $\phi$ & $\varphi$ & $\phi$ & $\varphi$ \\
\hline$\varphi$ & $q$ & $y$ & u & $\psi$ & $x$ & $\downarrow$ & $x$ & $\downarrow$ & $x$ & $\downarrow$ & $x$ \\
\hline 9 & $\mathrm{r}$ & $\phi$ & $\varphi$ & 8 & $\mathrm{f}$ & 8 & f & 8 & $\mathrm{f}$ & 8 & f \\
\hline$s$ & $\mathrm{~s}$ & $\psi$ & $x$ & & & & & & & & \\
\hline$T$ & $\mathrm{t}$ & 8 & $\mathrm{f}$ & & & & & & & & \\
\hline$y$ & u & & & & & & & & & & \\
\hline$x$ & $\dot{\mathrm{s}}$ & & & & & & & & & & \\
\hline$\phi$ & $\varphi$ & & & & & & & & & & \\
\hline$\psi$ & $x$ & & & & & & & & & & \\
\hline
\end{tabular}

Fig. 1. Serie alfabetiche documentate dagli alfabetari etruschi (da Bellelli e Benelli 2018, fig. 4). 
Per spiegare questi elementi apparentemente incongruenti (conservazione pedissequa e feticistica del modello greco in [1], alterazione sostanziale dello stesso in [2] e [3]) Prosdocimi ha elaborato la teoria del "corpus dottrinale", che si potrebbe riassumere come segue.

(1) La trasmissione della scrittura sarebbe avvenuta secondo le medesime procedure impiegate nell'apprendimento. In sostanza, da una parte ci sarebbe stato un "maestro", che recitava l'alfabeto come sequenza di valori fonici, dall'altra un allievo, che avrebbe appreso a memoria questa sequenza ritenendola nella sua interezza senza modificarla. Eventuali modifiche sarebbero intervenute solo in seguito, una volta che lo strumento scrittorio si fosse pienamente affermato, e si fosse avviata una riflessione sulla struttura fonematica della lingua tale da permettere uno o più adattamenti volti a piegare lo strumento alle necessità concrete di una resa più fedele dei suoni.

(2) "Corpus dottrinale" e "alfabeto teorico" non sono necessariamente coincidenti (e questa è la vera novità introdotta dalla teoria di Prosdocimi). In pratica, la recitazione della sequenza fonica avrebbe potuto preservare segni non solo abbandonati nella pratica scrittoria, ma anche nelle sequenze alfabetiche (alfabetari) materialmente realizzate.

Il pregio fondamentale di questo modello è quello di dare coerenza al processo di formazione dell'alfabeto etrusco, e di spiegare come mai le lettere "morte" potessero poi essere improvvisamente "resuscitate" in scritture di derivazione etrusca, quali il latino e il venetico; in questo senso è netta la sua superiorità ermeneutica rispetto alle ricostruzioni precedenti, che ipotizzavano alla base dellalfabeto etrusco un improbabile miscuglio di diversi alfabeti greci (e alla base degli alfabeti latino e venetico degli altrettanto improbabili miscugli di etrusco e di altro), e che possono ritenersi definitivamente accantonate. ${ }^{4} \mathrm{La}$ formazione di alfabeti per mescolanza consapevole di elementi di origine diversa è un fatto certamente attestato, ma in contesti storici e sociali abissalmente diversi rispetto all'Etruria del tardo VIII secolo a.C.; l'esempio più vicino è quello dell'alfabeto osco, una creazione fortemente artificiale che servì a marcare la nascita di una nuova identità etnico-politica, come mostra anche il suo uso preferenziale in un'epigrafia che è quasi esclusivamente di

4 Nonostante la loro periodica ricomparsa (p. es. Bagnasco Gianni 2008, 50), forse dovuta alla non immediatezza dello schema teorico di Prosdocimi. 
carattere pubblico, o comunque destinata a fruizione pubblica. ${ }^{5}$ Lepigrafia etrusca ha tutta un'altra storia, soprattutto nei suoi primi secoli. ${ }^{6}$

Nell'idea di Prosdocimi, il corpus dottrinale dei maestri greci (euboici) avrebbe incluso anche le due $x i$ (quella "morta", nella posizione del samekh fenicio e della $x i$ degli alfabeti "azzurri", e quella "viva", in coda, nel comparto dei segni complementari) e un suono di sibilante nella posizione dello tzade fenicio, privo di resa grafica in quanto assente dall'alfabeto (diverso rispetto al corpus dottrinale appreso dai maestri fenici e rimasto ipoteticamente inalterato nel sistema di apprendimento greco, fatta salva l'aggiunta in coda dei segni complementari). Il vuoto rappresentato da questo suono fantasma sarebbe stato sfruttato dagli Etruschi per inserire in quella posizione il san (che negli alfabetari greci è sempre alternativo a sigma, e nella posizione di sigma), in modo da venire incontro all'esigenza, indispensabile per una corretta notazione della lingua etrusca, di distinguere fra due sibilanti sorde. Questa idea di Prosdocimi, sia detto per inciso, nonostante il suo alto grado di ipoteticità (perché presuppone l'esistenza, in greco, di una sequenza mai documentata negli alfabetari), ${ }^{7}$ è infinitamente preferibile al mostro genealogico creato da Rudolf Wachter per gli alfabeti greci, nel tentativo di spiegare come mai un grafema (il san) che si ritiene nato dallo tzade fenicio (per la sua posizione nella sequenza etrusca) possa essere andato a finire nella posizione alfabetica dello shin (dove figura in tutti gli alfabetari greci). ${ }^{8}$ Questa ultima ricostru-

$5 \quad$ Cf. Benelli c. s. b.

6 Cf. Benelli c. s. c.

7 Dopo la pubblicazione del lavoro di Prosdocimi sono emersi due alfabetari greci che, apparentemente, documentano la presenza del san insieme al sigma, ponendo il primo esattamente dove si trova nella sequenza etrusca, cioè nella posizione dello tzade fenicio. Nessuno dei due è privo di problemi. Il primo è un testo rupestre dall'area di Barako o Keramoti, presso Vari, in Attica (Langdon 2005), rinvenuto in una zona ricca di iscrizioni rupestri che ancora attendono una pubblicazione, che permetterà di capire il contesto in cui è stato realizzato l'alfabetario. La sequenza contiene delle anomalie vistose quali la presenza di omografie imbarazzanti $(\gamma=\lambda$; san $=\mu)$ e di forme non riconducibili alla scrittura arcaica dell'Attica. L'autorevolezza dell'editore indurrebbe a escludere che si tratti di un falso (il sospetto è sempre presente per le iscrizioni rupestri, che, quanto meno nelle aree di mia diretta conoscenza, annoverano un numero considerevole di falsificazioni moderne), ma il suo preciso inquadramento nella storia alfabetica attica, e greca in generale, seguita a rimanere indefinibile. Il secondo esempio è quello delle famigerate tavolette di rame cosiddette "del Fayyum", per le quali un recente riesame sembrerebbe aver ribaltato il verdetto di falsità emesso dopo le prime analisi (Woodard 2014); le nuove indagini, tuttavia, sembrano aver incontrato solo un tiepido successo fra gli epigrafisti greci che, viste le stranezze dei documenti, tendono comunque a escluderli dalla ricerca, ritenendoli, se autentici, piuttosto non greci. 
zione è il riflesso di uno stato della ricerca anteriore al lavoro di Prosdocimi, che non faceva distinzione fra alfabeto teorico e corpus dottrinale. Ciò che accomuna le due posizioni è l'assunzione come principio universale, maturata proprio sulla scorta degli alfabetari etruschi, con la loro perpetuazione dei segni "morti", dell'idea che le sequenze alfabetiche fossero trasmesse come insiemi interi e inalterabili, se non eventualmente per aggiunta in coda (ma mai per sottrazione o modifica all'interno, possibili solo in un secondo tempo).

A tal proposito, non si può non notare già da subito (ma su ciò si tornerà più diffusamente in seguito) come l'elevazione a paradigma delle modalità di formazione dell'alfabeto etrusco è una scelta problematica, qualunque sia lapproccio metodologico preferito, se non altro perché in etrusco manca in modo pressoché completo il fenomeno di rifunzionalizzazione dei grafemi dell'alfabeto modello, che è invece prassi ordinaria in ogni altro caso di genesi alfabetica, mediterranea ${ }^{9}$ e non; già questo consiglierebbe cautela nel tentare di trarre principi generali da un evento che tutto sembra indicare come eccezionale.

A margine della discussione è importante notare che la presunzione di Prosdocimi di una comparsa tardiva del grafema etrusco $<\dot{s}>,{ }^{10}$ che è funzionale alla sua ricostruzione, non è corroborata dall'evidenza. La sua scarsa presenza nelle più antiche iscrizioni etrusche discende da due circostanze documentarie:

(1) l'epigrafia di area settentrionale ha nascita molto più tardiva rispetto a quella meridionale; in etrusco, il fonema /s/ (indicato da $<$ s $>$ al sud, $<$ ś $>$ al nord) è più comune di $/ \sigma /$ (probabilmente $/ S /$ );

(2) buona parte delle iscrizioni etrusche più antiche provengono da Cerveteri, dove, per quasi due secoli, la scrittura non distinse le due sibilanti sorde, usando indistintamente i grafemi disponibili (in genere il sigma).

In tali circostanze, è inevitabile che le sibilanti della prima documentazione epigrafica siano sempre dei sigma; non è così però se si prendono in considerazione le sigle. Già quando scriveva Prosdocimi si poteva constatare la presenza di $<$ ś $>$ nelle sigle del ripostiglio di S. Francesco a Bologna, il cui terminus ante quem (chiusura del grande recipiente contenente i bronzi) è l'inizio del VII secolo a.C. $;^{11}$ ora un $<$ ś> è documentato anche da una delle

9 Adiego 2018, 145-147, con bibliografia precedente.

10 Prosdocimi 1990, 213.

11 Sassatelli 1984, 155, nn. 36-37. 
più antiche sigle etrusche conosciute, quella incisa su un vaso di legno dalla Tomba del Guerriero di Tarquinia, che rimanda a una datazione attorno al 730 a.C. ${ }^{12}$ Questa circostanza, in sé, non inficia la ricostruzione di Prosdocimi, limitandosi semplicemente a spingere indietro gli stadi formativi della scrittura etrusca in fase pre-epigrafica, un periodo nebuloso (per l'ovvia mancanza di documentazione diretta) ma certamente di durata non irrilevante. ${ }^{13}$

2. Le tappe di evoluzione della struttura (teorica) dellalfabeto etrusco sono state complicate dalla scoperta di ET Cm 9.9 (fig. 2), che testimonierebbe la soppressione dei segni "morti" nella sequenza già a una quota cronologica molto alta (terzo quarto del VII secolo a.C.), configurando la possibilità dell'esistenza di tradizioni alfabetiche parallele, una maggioritaria e una secondaria. ${ }^{14}$ La lettura presenta alcuni problemi, a cominciare dal riconoscimento del segno centrale come un gamma ispirato al tipo a uncino (ma che assume piuttosto le sembianze di una croce asimmetrica). Questo tipo di segno ricorre anche in un altro testo, CIE $6669=E T$ Ve 9.5 (fig. 3), più antico di qualche decennio e proveniente da Veio, la cui identificazione come alfabetario sembra più solida. Questi documenti sono stati molto valorizzati da Giovanni Colonna, nel tentativo di identificare un sistema scrittorio alternativo a quelli epigraficamente noti, documentato solo in modo estremamente frammentario, che sarebbe alla base anche dell' iscrizione di Osteria dell'Osa. ${ }^{15}$ Lo stato dell'evidenza suggerisce qualche cautela, ma non cè dubbio che, se la presenza di una forma di scrittura (etrusca? o estesa anche ad altre lingue centro-italiche?) alternativa rispetto a quella comunemente utilizzata venisse ulteriormente confermata da nuove scoperte, si tratterebbe di un fattore di cui tener conto.

\section{Benelli 2013.}

13 Benelli c. s. a.

14 Fenomeno analogo potrebbe essere testimoniato, allestremo opposto del mondo etruscofono, da REE 80, 1 (Bologna), ancora più antica, la cui interpretazione resta comunque incerta. Su questo (possibile) alfabetario si veda infra, $₫ 6$, punto 5 ("soluzione b").

15 Colonna 2016. 
Enrico Benelli

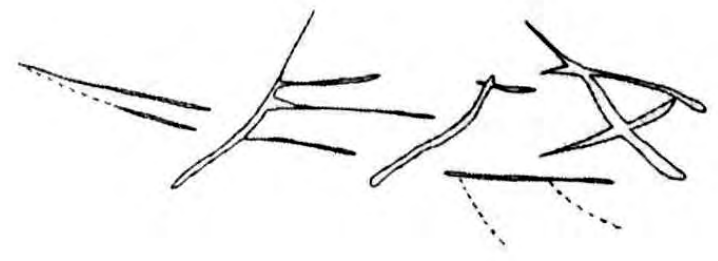

Fig. 2. Possibile alfabetario da Pontecagnano (da REE 71, 63).

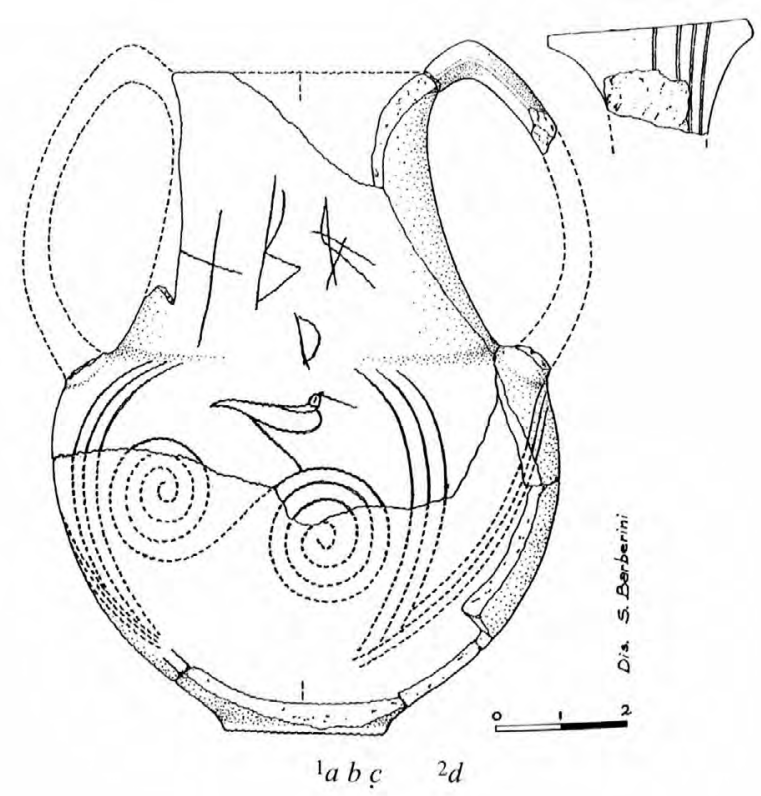

Fig. 3. Possibile alfabetario da Veio (da CIE 6669).

È però nel settore greco che si sono avute le scoperte più rilevanti degli ultimi decenni, che hanno contribuito a rileggere le fasi formative della scrittura. ${ }^{16}$ Il primo gruppo di materiali è venuto alla luce ad Eretria; ${ }^{17}$ fra questi si segnala un frammento di alfabetario che comprende la $\xi$ "azzurra" (posizionata fra $v$ e o), nella medesima forma a finestrella nota negli alfabetari etruschi (fig. 4). Questa sorprendente attestazione è apparsa una conferma dell'idea avanzata da Prosdocimi che il repertorio dei segni portati dagli Euboici

16 Per maggiore dettaglio sullo stato delle conoscenze si rimanda anche al contributo di Giovanni Boffa in questa sede.

17 Kenzelmann-Pfyffer, Theurillat e Verdan 2005. 
in Etruria andasse molto al di là di ciò che normalmente indichiamo come "alfabeti euboici", e che in fondo non era necessario postulare nessun innesto di altra origine al momento della formazione della scrittura etrusca. Ma cè di più. In queste iscrizioni di Eretria (e in primis proprio nel citato alfabetario) riappare, sia pure in posizione minoritaria, il pi a uncino già documentato a Pitecusa, che postula un gamma di forma diversa (per il principio delle coppie legate, le cui forme si influenzano a vicenda per evitare i rischi di omografia). Pur mancando tuttora un'attestazione positiva, è probabile che questo gamma fosse proprio quello lunato attestato nelle iscrizioni etrusche fin dal più antico documento epigrafico (CIE $10159=E T$ Ta 3.1), e che compare nellepigrafia euboica solo un secolo più tardi. Anche questo era già stato ipotizzato da Prosdocimi ${ }^{18}$ prima della scoperta dei nuovi documenti eretriesi.
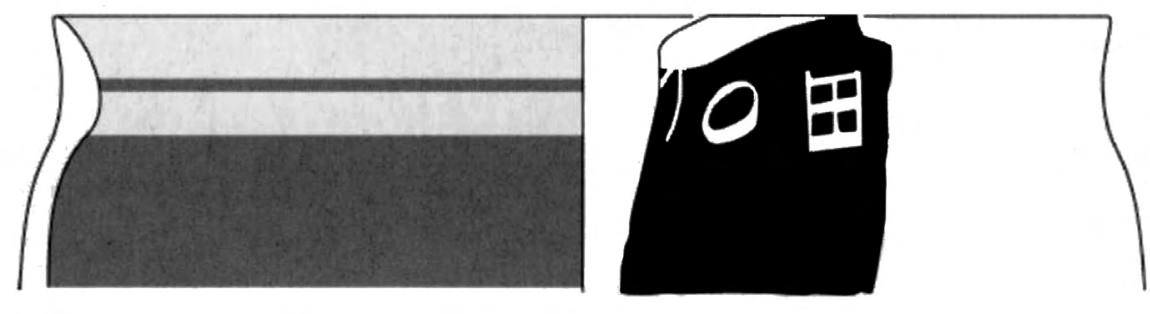

Fig. 4. Frammento di alfabetario da Eretria (da Kenzelmann-Pfyffer, Theurillat e Verdan 2005, 60, n. 3).

In tempi più recenti a Metone è emerso un nuovo importante nucleo di iscrizioni greche di altissima antichità, per lo più euboiche; la rilevanza del ritrovamento ha stimolato un vivace dibattito che ha coinvolto anche un ripensamento dei processi genetici delle stesse scritture greche. ${ }^{19}$ Uno degli aspetti più rilevanti dell'accrescimento della documentazione epigrafica risalente all'VIII secolo è la constatazione che il medesimo ambiente culturale (euboico) poteva dare vita a forme grafiche non solo diverse, ma anche contraddittorie: e questo non solo nel settore delle coppie legate (gamma - pi, per esempio), ma anche in quello dei segni complementari. Uno dei nuovi testi di Metone, infatti, tramanda, in un contesto alfabetico euboico (probabilmente eretriese), un segno a croce che è stato proposto di leggere $\chi$ (anche se non mancano voci discordanti), più o meno alla medesima quota cronologica in

19 Strauss Clay, Malkin e Tzifopoulos 2017. 
cui compare a Pitecusa con l'atteso valore $\xi .{ }^{20}$ Se si mette in parallelo questa attestazione con la $\xi$ a finestrella di Eretria, non si può che concludere che nelle scritture euboiche, a livello di VIII secolo, la distribuzione dei valori attribuiti ai segni era tutt'altro che definitiva; se la lettura proposta dagli editori per la nuova iscrizione di Metone è corretta (il che è ancora sub iudice), non si può che concludere che lopzione "rossa", poi codificatasi nel secolo successivo, era solo una di quelle possibili.

3. Prendendo in considerazione l'insieme delle scoperte degli ultimi 25 anni, ce nè in abbondanza per dire che la teoria del "corpus dottrinale" di Prosdocimi ne esce non solo brillantemente confermata, ma è anche l'unico modo per poter rendere conto di tutti i fenomeni osservabili nella più antica documentazione epigrafica, escludendo in modo ormai definitivo tutte le ricostruzioni (come la genealogia di Wacther già citata) basate su una lettura troppo formale delle strutture degli alfabeti storici. È ormai evidente che, al livello cronologico della trasmissione agli Etruschi della scrittura alfabetica greca, quest'ultima non era ancora organizzata nei classici alfabeti poliadici che siamo abituati a vedere nei manuali, ma consisteva di un insieme latamente organizzato (limitatamente alla sua sequenza) di grafemi alternativi e potenzialmente contraddittori fra i quali era possibile operare scelte diverse. Di un corpus dottrinale, appunto.

Quello che però resta da definire è se l'idea che una scrittura sia rappresentata da un corpus dottrinale non coincidente con quello che Prosdocimi chiama "alfabeto teorico" o "alfabeto princeps" sia sempre e universalmente valida, oppure no. La risposta, probabilmente, è negativa. È verosimile che questo stato di fluidità non possa funzionare come categoria interpretativa universale nel tempo e nello spazio; nello stesso mondo greco, dopo l'VIII secolo a. C., si formano in modo piuttosto stabile degli alfabeti poliadici dal forte valore identitario. Proprio il fatto che la loro formazione sia successiva a un periodo di fluidità ne accresce il significato come componente visibile di un'affermazione identitaria.

Lo stesso deve essere avvenuto nella scrittura etrusca almeno al momento del passaggio dalla prima alla seconda fase degli alfabetari (ma probabilmente anche prima: su ciò si veda infra, $₫ 5$ ), che non a caso coincide quasi perfettamente con l'attività della cosiddetta "scuola veiente", la prima tradizione

20 Janko 2017, 157, con riferimenti, e Méndez Dosuna 2017, 249-258, per una lettura discordante rispetto a quella preferita dagli editori (segno a croce $=$ coerente $\xi$ "rossa"). 
scribale etrusca chiaramente identificabile, che non a caso si manifestò proprio a Veio, dove l'introduzione di severe limitazioni al lusso funerario, sullo scorcio del VII secolo, indusse a traslare verso il santuario le forme principali di ostentazione aristocratica, debitamente dotate di corredo epigrafico. Tutto il processo di riflessione sulla scrittura compiuto da questa scuola scribale sarebbe inimmaginabile senza una contestuale decantazione di un alfabeto ormai compiutamente definito. Che la riforma dell'alfabeto teorico etrusco abbia probabile matrice veiente è suggerito non solo dalla documentazione superstite (che può essere distorta da fattori casuali), ma anche dall'aspetto degli alfabetari della seconda fase che, nella loro redazione meridionale, indicano una concezione della scrittura che resta comunque fortemente influenzata da fattori teorici più che dall'uso pratico, in quanto (a) continuano a conservare la notazione ridondante dei tre grafemi per $/ \mathrm{k} /$, di fatto sempre più completamente abbandonata, così come il grafema $<\dot{\mathrm{s}}\rangle$, di uso molto limitato nel tempo e nello spazio (e tipico proprio della scuola veiente, fra fine VII e prima metà del VI secolo), (b) continuano a rifiutare pervicacemente l'inserimento di $\langle\mathrm{f}\rangle$ in coda alla sequenza nonostante l'introduzione nell'uso epigrafico già sullo scorcio del VII secolo (con la notevole eccezione, non a caso, dell'area cerite-veiente, dove la resa con il digramma $\langle\mathrm{vh}\rangle \mid\langle\mathrm{hv}\rangle$ continua a persistere fino al VI secolo inoltrato). Una concezione che, quindi, è evidentemente risultato di una codificazione che deriva da una riflessione teorica sulla scrittura, non necessariamente coincidente in toto con la pratica scrittoria quotidiana.

Se tutto questo è vero, ne consegue che l'idea di una trasmissione della scrittura sotto forma di corpus dottrinale, e non di alfabeto princeps, nel senso teorizzato da Prosdocimi, è sì l'unica lettura possibile dei fenomeni formativi delle prime scritture greche, della trasmissione alletrusco, e da questo ad altre realtà italiche, ma è comunque uno strato fenomenologico limitato nel tempo, e destinato ad essere sostituito da altre forme di concezione dellespressione scritta con il passare dei secoli. Lo stadio del corpus dottrinale è una realtà contingente e non universale, legata a specifiche situazioni storiche, sociali e culturali. È riscontrabile nel mondo greco dell'VIII secolo, quando evidentemente il valore identitario della scrittura doveva essere ancora limitato (tanto è vero che, a quanto sembra, ad Eretria un greco scrisse la propria lingua addirittura in alfabeto fenicio), ${ }^{21}$ ma non più nel VII, almeno nella maggior parte delle realtà politiche e culturali, lì dove l'uso di determinate forme

21 Kenzelmann-Pfyffer, Theurillat e Verdan 2005, 76-77 (n. 66). 
(e la contestuale deliberata esclusione di altre) acquisì forza di identificatore, anche in senso contrastivo rispetto al mondo circostante. Questa prospettiva, tra l'altro, aiuta anche a capire quei rari casi, soprattutto di ambiente coloniale, nei quali sembra persistere ancora entro il VII secolo qualche contraddizione nel tipo di scrittura utilizzata; anche in questo caso le spiegazioni spesso macchinose che si sono raggiunte utilizzando approcci di tipo formalistico, che presuppongono che gli alfabeti greci arcaici fossero realtà chiuse e impenetrabili, evaporano immediatamente se solo si pensi a un più lungo processo di decantazione di una scrittura stabile, provvista di valore identitario, dal più vasto corpus dottrinale primigenio.

Lo stadio di corpus dottrinale fluido delle scritture greche al momento della trasmissione alletrusco è provato anche da un altro elemento scarsamente valorizzato nella tradizione degli studi, ossia l'andamento del sigma a tre tratti, che nella scrittura etrusca è preferenzialmente retrogrado. L'origine di questa forma, probabilmente, va ricercata in una terza soluzione della potenziale omografia sigma/iota (alternativa rispetto alle altre due normalmente preferite negli alfabeti greci dal VII secolo in poi) documentata dalla sola iscrizione delloinochoe del Dipylon (fig. 5), testo attico di alta antichità caratterizzato da tale originalità nelle proprie forme grafiche da essere una testimonianza evidente del fatto che la decantazione degli alfabeti poliadici era ancora al di là da venire. Anche questa opzione doveva essere presente nel multiforme corpus dottrinale greco giunto agli Etruschi.

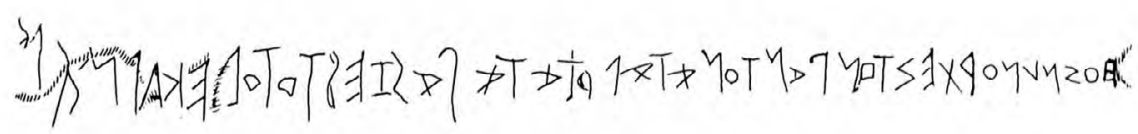

Fig. 5. Iscrizione dell'oinochoe del Dipylon (da Guaarducci, 42, fig.) 
4. La teoria del corpus dottrinale di Prosdocimi, così come le spiegazioni vetero-formalistiche che ancora dominano molta bibliografia non italiana, condividono però un punto debole. In entrambi gli approcci, la scrittura sarebbe una sorta di organismo dotato di vita propria, del tutto indipendente dalla volontà di chi riceve questo strumento tentando di adattarlo, in modo più o meno efficace, alle proprie necessità. Questo è anche un effetto del peso che ha avuto la (cospicua) documentazione degli alfabetari etruschi nella discussione in ambito classicistico (non solo etruscologico); la lunga persistenza delle lettere "morte" ha dato un'idea degli Etruschi come sopraffatti da qualcosa di ieratico e inalterabile, al quale avrebbero risposto con un ossequio goffo e provinciale, da barbari quali erano. È soprattutto da questa impressione che discende l'applicazione alla trasmissione della scrittura del modello "maestro-allievo", con il secondo in posizione subordinata rispetto al primo, privato della capacità di prendere qualunque iniziativa autonoma. In questo modello di trasmissione, chi riceve la scrittura in qualche modo la "subisce", senza avere la possibilità di modificare alcunché; eventuali modifiche riscontrabili nellevidenza sarebbero nient'altro che effetto di incomprensione del modello.

L'utilissima raccolta di Daniels e Bright 1996, pur essendo per forza di cose cursoria e, in qualche caso, superata, ${ }^{22}$ permette di avere una panoramica globale della grande maggioranza dei sistemi scrittori del mondo. Da questo emerge come, lì dove esista documentazione sufficiente, il fattore di agency è sempre determinante nella nascita dei sistemi scrittori; chi ne acquisisce uno, opera delle scelte che hanno un senso culturale e identitario che può essere molto marcato, e che può anche essere un fattore più importante rispetto alla corretta resa grafica dei fonemi della lingua che si intende scrivere. Il fatto è particolarmente evidente in tutte quelle scritture che acquisiscono, sul piano visivo/formale, aspetti dichiaratamente contrastivi nei confronti di altri sistemi scrittori con i quali sono in contatto diretto. Un esempio classico è quello dell'alfabeto armeno; ma anche quello georgiano potrebbe aver avuto una genesi simile; ${ }^{23}$ soprattutto nel caso dell'armeno, la volontà di distinguersi dal greco bizantino è palese, soprattutto lì dove grafemi apparentemente simili a quelli greci sono usati per valori fone(ma)tici completamente diversi.

22 Per il mondo classico, per esempio, lo stadio di comprensione delle scritture anatoliche riflesso nell'opera è ancora molto parziale. Per un aggiornamento complessivo si veda Adiego 2018.

23 Sanjian 1996; Holisky 1996. 
Casi simili si trovano anche in tutti quei fenomeni di genesi recente di sistemi scrittori in aree sottoposte alla pressione di culture pervasivamente litterate, spesso (ma non sempre) coloniali, come il Nord America, l'Africa occidentale, l'India centrale e il Sud-Est asiatico. ${ }^{24}$ In questi ultimi casi, il ricorrere di fattori comuni anche in situazioni culturali molto distanti fra loro, e caratterizzate da esigenze politico-sociali anche piuttosto diverse, ${ }^{25}$ fa pensare che l'individuazione di una scrittura visivamente diversa da qualunque altra fosse senzaltro un elemento identitario. Ritornando a realtà più vicine all'Italia preromana, un'uguale volontà di affermazione di un'identità contrastiva potrebbe essere alla base delle scritture libico-berbere nel loro controverso rapporto con il modello (probabilmente) fenicio; ${ }^{26}$ e lo stesso deve valere per la singolare scrittura sidetica. ${ }^{27} \mathrm{~A}$ ben vedere, anche un fenomeno familiare ai classicisti come la decantazione delle scritture greche arcaiche da un corpus dottrinale condiviso è segno indicativo della volontà di un’affermazione identitaria (locale) tramite opzioni alfabetiche divergenti.

Naturalmente, se sono proprio le scelte dichiaratamente contrastive a rendere evidente, per chi osserva il fenomeno a posteriori, il valore identitario di un sistema scrittorio, valore altrettanto forte deve essere probabilmente attribuito anche alle scelte non contrastive, che, per loro stessa natura, sono più difficili da isolare nel quadro documentario. Come che sia, il fattore di agency nella formazione di un nuovo sistema scrittorio riappare in modo talmente chiaro in contesti geografici, cronologici e culturali tanto diversi da poter essere considerato una costante. Che solo gli Etruschi fossero incapaci di comportarsi nello stesso modo sarebbe una circostanza alquanto improbabile.

Alle origini della scrittura etrusca (e non solo etrusca), quindi, deve postularsi necessariamente l'esistenza di una consapevole agency, responsabile

24 Si veda la parte IX ('Scripts invented in modern times') in Daniels e Bright 1996, 577-624; la situazione è però molto più complessa di quella riassunta nel manuale. Per l'Africa occidentale $c f$. da ultimo Kelly 2018a, per il sud-est asiatico Kelly 2018b, per l'India Guillaume-Pey 2018, ai quali si rimanda per la bibliografia precedente.

25 In Africa e in Nord America la reazione è alla presenza europea, laddove nel Sud-Est asiatico, almeno nelle leggende connesse alla rivelazione della scrittura, predomina una volontà contrastiva nei confronti della Cina. In India centrale, le pressioni coloniali e quelle delle civiltà litterate indiane dominanti si annullano a vicenda, portando alla scelta di una "terza via". L'origine delle nuove scritture è spesso attribuita a ispirazione o epifania divina (Africa, Sud-Est asiatico, Ho dell'India centrale), ma vi sono casi di genesi del tutto laica (Nord America, Sorang Sompeng dell'India centrale). 
di ogni scelta, sia di carattere positivo (contrastivo) che negativo (non contrastivo). In questo senso, e non come risultato di inettitudine o sudditanza culturale degli scribi etruschi, va letto anche il rifiuto quasi totale della rifunzionalizzazione dei grafemi, al contrario di quello che succede nella maggior parte delle formazioni di scritture alfabetiche, anche nello stesso mondo mediterraneo, e più o meno alla medesima quota cronologica in cui si forma la scrittura etrusca ( si vedano, per esempio, le scritture anatoliche, e soprattutto il cario, ma anche, nella stessa penisola italiana, il sabino e la sua filiazione "sudpicena").

5. Il repertorio di Daniels e Bright 1996 permette di andare a cercare dei paragoni per quello che seguita a restare il fenomeno più singolare della scrittura etrusca, ossia la persistenza, a livello di alfabeto teorico documentato negli alfabetari, delle lettere "morte". La singolarità di questa persistenza si misura nel fatto che, se è vero che è certo possibile realizzare iscrizioni, ivi compresi gli alfabetari, ancora ad uno stadio di corpus dottrinale, la presenza di un numero abbastanza cospicuo di alfabetari che mostrano caratteristiche costanti indica che il passaggio dal corpus dottrinale (per sua natura fluido e non privo di potenziali contraddizioni interne) all'alfabeto teorico stabile è, per forza di cose, completato. Questa tappa, in etrusco, sembra compiersi quanto meno entro la metà del VII secolo; è interessante, in questo senso, che gli alfabetari aberranti, sia quelli più profondamente anomali (la scrittura alternativa ipotizzata da Colonna), sia quello solo parzialmente deviante dell'effimera riforma "euboizzante" (espressa dall'alfabetario di Marsiliana e dalla serie dei kyathoi cosiddetta "Caere-Vetulonia-Monteriggioni"), ${ }^{28}$ che è in contrasto con un uso diverso, e pressoché universale, affermatosi già da almeno una generazione, siano anche i più antichi. Tutto questo fa pensare che già verso il 650 a.C., ben prima dell'attività della "scuola veiente" alla quale si è accennato sopra, il passaggio è ormai avvenuto in maniera irreversibile; dal corpus dottrinale si è decantato l'alfabeto teorico. Quindi, la questione non è tanto quella di avere dei segni "morti" nel corpus dottrinale (che sarebbe poco sorprendente), ma di ritenerli nell'alfabeto teorico.

Ora, andando a scorrere il repertorio di Daniels e Bright 1996 si scopre che questo fenomeno, quando accade (il che è piuttosto raro), ha sempre

28 Sulla riforma euboizzante Bellelli e Benelli 2018, 56, con bibliografia precedente; sulla serie dei kyathoi v. ora Cappuccini 2018 (e Tuck e Wallace 2018 per un ulteriore frammento da Murlo). 
delle motivazioni. Al netto dei casi in cui i segni sono "morti" per questioni di evoluzione fonetica della lingua (ma in origine dovevano essere "vivi"), la documentazione di sistemi scrittori che includono grafemi apparentemente inutili si riduce a due gruppi principali: (a) le scritture arabe usate per le lingue dell'Asia centrale e (b) le scritture di derivazione indiana del Sud-Est asiatico e del Tibet. ${ }^{29}$ In entrambi i casi ladozione di questi sistemi scrittori è avvenuta al seguito dell'espansione di religioni basate sui testi scritti; se nel Sud-Est asiatico la scrittura sillabica di derivazione indiana non incontrò alcuna preesistenza, in Asia centrale le cose stavano diversamente. Il mondo persiano, ad esempio, aveva una lunga tradizione di scritture, che furono soppiantate completamente da quella araba, portata dalla rapida espansione della religione islamica; l'adozione della nuova scrittura dovette avere un valore enorme di scelta culturale, anche per la sua incapacità di rendere con precisione la lingua persiana.

La diffusione di questi sistemi scrittori, infatti, comportò degli adattamenti macchinosi e mai realmente soddifacenti; un alfabeto consonantico come quello arabo, ad esempio, non è particolarmente adatto a rendere lingue indoeuropee come il Farsi o l'Urdu, e ancora meno per lingue turche come il Turco Ottomano o l'Uiguro (almeno fino all'introduzione delle riforme grafiche del 1951/54 e del 1983). Ancora più problematica è la situazione del SudEst asiatico, dove la scrittura di origine indiana, che adotta le sillabe aperte come unità di base, deve venir piegata a lingue completamente diverse, con frequenti sillabe chiuse, a volte anche con toni vocalici, giungendo a esiti di una complessità sconcertante, con una proliferazione impressionante di diacritici. In entrambi i casi, quindi, il prestigio del modello, inestricabilmente congiunto con il fattore religioso, ha condotto all'adozione di sistemi scrittori profondamente inadeguati a rendere la realtà fone(ma)tica di quelle lingue. Se non sapessimo quali potenti motivazioni culturali hanno sostenuto queste scelte linguisticamente irrazionali, non potremmo mai capirne il motivo.

Questi due insiemi di sistemi scrittori condividono la presenza di lettere "morte", che, nel caso delle scritture di derivazione indiana del Tibet e del

29 Grafemi apparentemente ridondanti sono presenti anche in altri sistemi scrittori, anche se in modo meno pervasivo; in linea di massima, si può supporre che le motivazioni culturali non siano dissimili da quelle evidenziate in seguito. Esempi evidenti sono la prima scrittura cirillica (con i grafemi greci: Cubberley 1996), e la scrittura dell'amarico, in rapporto con il ge'ez (Menuta 2016). 
Sud-Est asiatico, possono ammontare a intere serie. ${ }^{30} \mathrm{Il}$ motivo della loro presenza è piuttosto trasparente: usare un'unica scrittura sia per la propria lingua che per la lingua (del tutto diversa) dei testi sacri. Si tratta, in sostanza, di scritture per le quali l'interoperabilità (con una lingua sacra) è più importante della corretta resa fonematica e della razionalità di impiego. ${ }^{31}$

6. Nel mondo classico, naturalmente, non esisteva una religione basata su un corpus di testi scritti da trasmettere insieme alla scrittura, dalla Grecia verso l'Italia o l'Asia minore. Il persistere di segni “morti” nell'alfabeto etrusco (e solo nell'alfabeto etrusco), quindi, deve avere unaltra motivazione, potenzialmente potente come il fattore religioso in tempi più moderni. Prima di andare a cercare questa motivazione, è forse necessario ripercorrere in modo schematico le tappe di formazione della scrittura etrusca viste fino a questo punto.

(1) In un primo momento (VIII secolo a.C.), quando nasce l'idea di usare la scrittura greca (singolare!) per rendere l'Etrusco, questa si trova ancora allo stato fluido di corpus dottrinale. In tale stato viene recepita dagli Etruschi, rendendo possibili esiti grafici parzialmente contraddittori, come accadeva anche nel modello greco.

(2) Nel mondo greco, a partire dagli inizi del VII secolo, si passa dallo stadio di corpus dottrinale a quello degli alfabeti locali, contraddistinti da un complesso gioco di opposizioni e corrispondenze, che riflettono volontà di affermazioni identitarie più o meno oppositive nei confronti delle realtà politiche circostanti. Si deve ritenere verosimile che questo processo non sia avvenuto contemporaneamente in tutto il mondo greco, ma possa aver avuto tempi diversi a seconda delle diverse realtà politiche, sociali e culturali. Solo in questo modo si può rendere conto del permanere di fatti grafici apparentemente contraddittori in alcune regioni. ${ }^{32}$

30 Nella leggenda mitistorica sull'origine della scrittura tibetana si presuppone una reintroduzione posteriore dei segni per i fonemi del sanscrito; ma tutta la storia va letta nel quadro di unaffermazione identitaria posteriore: Van Schaik 2011.

31 Per quanto riguarda l'uiguro, la riforma, promossa dalle autorità della Repubblica Popolare, nel modificare la scrittura araba in modo che potesse rendere correttamente la fonologia della lingua, non casualmente rimosse anche tutti i segni ridondanti, usati per la resa delle parole arabe, facendo cessare la piena interoperabilità della scrittura uigura per le due lingue.

32 In epoca posteriore all'VIII secolo le apparenti contraddizioni fra più modelli alfabetici conviventi sono particolarmente evidenti in ambiente coloniale; tuttavia non mancano casi dalla stessa Grecia metropolitana. Un esempio del permanere di sistemi scrittori 
(3) Quasi contemporaneamente accade lo stesso anche nel mondo etrusco; il processo è sicuramente concluso entro la metà del VII secolo, con la creazione del primo alfabeto teorico stabile (alfabetari della prima fase). ${ }^{33}$

(4) La trasmissione al latino potrebbe essere avvenuta quando l'alfabeto etrusco era ancora allo stadio di corpus dottrinale; sono acquisiti, in corretta posizione, $\langle\mathrm{b}\rangle,\langle\mathrm{d}\rangle,<\mathrm{o}\rangle,<\mathrm{x}\rangle$ (con valore originario del greco "rosso", evidentemente ancora mantenuto nella recitazione della sequenza). La mancata differenziazione dei segni per / $/ \mathrm{e} / \mathrm{g} /$ è forse esito della pronuncia come $/ \mathrm{k} /$ del grafema di gamma da parte dei maestri etruschi. È ritenuta la $<z>$, che ha inizialmente un qualche sporadico impiego in latino, ma, probabilmente, sono consapevolmente espunti fin da subito il san, la $\xi / s a m e k h$ e i grafemi delle aspirate $(\theta, \varphi, \chi$ : questi ultimi due poi recuperati come segni numerali), anche se il più antico alfabetario latino è decisamente più $\operatorname{tardo}^{34} \mathrm{e}$, in questo senso, non aiuta molto.

(5) La trasmissione della scrittura etrusca verso nord è una questione meno chiara.

(a) Comune agli alfabeti settentrionali più antichi (leponzio e venetico) è la presenza di $\langle$ o $>$ e l'assenza $\mathrm{di}<\mathrm{b}\rangle,\langle\mathrm{d}\rangle$ e delle due $\langle\xi\rangle$. Al momento, non esistono alfabetari leponzi completi che possano testimoniare lordine delle lettere; la documentazione epigrafica mostra che la nascita di questa scrittu-

alternativi potrebbe essere l'alfabetario di Keramoti (cf. supra, nota 7), se la cronologia proposta dalleditore (VI secolo) cogliesse nel vero (ma datare un'iscrizione rupestre è, per sua stessa natura, problematico). La testimonianza forse più evidente di questo fenomeno, però, è la presenza di iscrizioni in alfabeto "non corinzio" su alcuni prodotti ceramici di manifattura corinzia (Wachter 2001, 30-33), per le quali sono state proposte varie spiegazioni; in particolare, per l'opera più insigne della ceramografia protocorinzia, lolpe Chigi, la critica recente si divide fra chi sostiene unorigine non corinzia del pittore (D'Acunto 2013, 131-137, con bibliografia precedente) e chi pensa che lo stesso, pur essendo corinzio, abbia piuttosto voluto riprodurre una pittura, forse celebre, realizzata, con tanto di didascalie, da un artista non corinzio (Maras 2014a, 148-149, con bibliografia precedente). Forse tutte queste spiegazioni non sono necessarie se immaginiamo che la decantazione di un alfabeto "corinzio" come elemento identitario sia stato un fenomeno relativamente tardivo, e, in caso, inizialmente non del tutto cogente per la pratica epigrafica non formale.

33 Il posizionamento di san (etrusco) nel luogo dello tzade fenicio è documentato a partire da questo momento. Resta incerta la sua genesi: presenza di un suono nel corpus dottrinale greco non corrispondente a un segno (e eventualmente documentato dal solo alfabetario di Barako/Keramoti) come ipotizzato da Prosdocimi, o deliberato atto di agency (il cui intervento è sotteso nella stesa creazione di una scrittura stabile) che risente, ad esempio della conoscenza indipendente dellarchetipo fenicio? 
ra dovette avvenire entro il VII secolo, molto prima di quanto si credeva in passato, quindi in un momento in cui era già avvenuta la stabilizzazione di un alfabeto teorico etrusco, che però conservava ancora almeno due grafemi $(<\mathrm{b}>\mathrm{e}<\mathrm{d}>)$ che avrebbero potuto essere utili. A complicare la situazione è la presenza, in una sola iscrizione leponzia non priva di stranezze, del grafema $<\mathrm{q}>$. Il venetico è documentato solo a partire dal VI secolo; lordine delle lettere è noto grazie ai numerosi alfabetari, che riflettono una struttura che separava le consonanti dalle vocali, forse contingente alla specifica classe documentale. ${ }^{35}$ L'assenza di $\langle\mathrm{c}\rangle$, sia nel leponzio che nel venetico è coerente con l'idea che il modello etrusco che ispirò questi sistemi scrittori doveva essere di area settentrionale, ma la retrodatazione dell'origine della scrittura leponzia confligge con il fatto che l'unico alfabetario etrusco-settentrionale appartenente alla prima fase (ET Vt 9.1, dello scorcio del VII secolo), riproduce la medesima sequenza nota da quelli meridionali, ossia l'alfabeto teorico completo antecedente la riforma della fine del secolo.

(b) Oltre a ciò, vi sono altri problemi. Espunzione di $\langle\mathrm{c}\rangle$ per adozione, negli alfabetari, della realtà fattuale della scrittura settentrionale (invece dell'alfabeto teorico), comporterebbe anche espunzione di $<q>$ (oltre che di $<0>$ ), che è però presente (sia pure come unicum) in leponzio arcaico. ${ }^{36} \mathrm{Il} \mathrm{sig-}$ $m a$, sia in venetico che in leponzio, è resa coerente di /s/, fonema che l'etrusco del nord scriveva piuttosto con san.

(c) L'unico modo per giustificare tutte queste contraddizioni è postulare un alto livello di agency da parte delle civiltà dell'Italia settentrionale che ricevettero l'alfabeto dagli Etruschi. Questo può essere facilmente accettabile per il Veneto, teatro di un culto (quanto meno a Este) della scrittura in quanto tale; i Veneti antichi seppero rimodellare, anche strutturalmente, la base concettuale dell'alfabeto etrusco, attingendo (certo consapevolmente) a modelli diversi, compresa la comparsa tardiva dell'interpunzione sillabica, posteriore al suo abbandono da parte della scuola veiente che pure la aveva inventata (e parallela, invece, alla sua introduzione nell'etrusco della Campania settentrionale, fatto che deve far riflettere sulla capacità di circolazione dei modelli). La scrittura leponzia, al contrario, mostra delle instabilità croniche, risolte in

35 Lo stato delle conoscenze sulla scrittura leponzia è riassunto in Maras 2014b; per il venetico Marinetti 2013.

36 E non è presente nell'alfabetario chiusino (tardo-arcaico, e già riformato) ET Cl 9.2 (pace Rix). 
gran parte solo in un momento piuttosto tardo, che sembrerebbero indicare un grado di fluidità ben diverso rispetto al Veneto.

(d) A prima vista verrebbe da pensare:

(soluzione a) che il motivo del rifiuto $\mathrm{di}<\mathrm{b}>$ e $<\mathrm{d}>$ stia nel medesimo fatto che causò la confusione fra / k/ e /g/ in latino: la pronuncia /k/ del gamma da parte degli Etruschi. A differenza di quella latina, la scrittura venetica avrebbe deciso di attuare una soluzione coerente per la resa delle sonore, rifunzionalizzando $\langle\theta\rangle,\langle\varphi\rangle,\langle\chi\rangle$ e $\langle z\rangle$; anche in leponzio vi sono tracce di una soluzione analoga, che però è ben lungi dall'essere univoca. In sostanza, sembra che fino al IV secolo la scrittura leponzia si comporti come un corpus dottrinale, ${ }^{37}$ a differenza di quella venetica, che, già fin dalle prime testimonianze epigrafiche, ha piena stabilità di alfabeto teorico ormai definito. Ma se la scrittura leponzia mantiene la condizione fluida di un corpus dottrinale, come giustificare l'espunzione $\mathrm{di}\langle\mathrm{b}\rangle,\langle\mathrm{c}\rangle \mathrm{e}<\mathrm{d}\rangle$ (potenzialmente utili, a differenza delle due $\xi$ ), che alla quota cronologica della sua nascita erano presenti nel modello teorico etrusco? A questo punto si può proporre un'altra spiegazione:

(soluzione b), alternativa rispetto alla precedente, e puramente congetturale: che alla quota cronologica del pieno VII secolo (punto 3, supra), si siano decantate $d u e$ sequenze teoriche, una meridionale e una settentrionale, con un confine non coincidente con le aree ortografiche; l'alfabetario di Monteriggioni (ET Vt 9.1), dove l'ortografia è settentrionale, sarebbe il rappresentante più settentrionale (a noi noto) dell'area alfabetica meridionale, mentre più a nord (Valdarno? Area padana?) si sarebbe decantato un altro alfabeto teorico, senza $<$ b $>,<\mathrm{c}>$ e $<$ d $>$, ma con $<$ o $>$ e $<$ q $>$, alla base del fluido corpus dottrinale leponzio e del rigoroso alfabeto venetico. Fino a nuove scoperte questa idea è destinata a restare nel campo delle pure ipotesi di lavoro. ${ }^{38}$

(6) La trasmissione al sabino implica invece un elevato livello di agency, se non altro perché i Sabini, unici in Italia centro-settentrionale, scelsero nel corpus dottrinale greco(/etrusco) la $<\mathrm{m}>$ a quattro tratti (che, incidental-

37 Sullorigine della scrittura leponzia sotto forma di corpus dottrinale, unica spiegazione possibile per la sua instabilità, $c f$. Solinas 2015 ; sul forte valore identitario collegato con la fase tarda (stabile) della scrittura: Franc 2018.

38 L'unica possibile testimonianza dell'esistenza di questo secondo alfabeto teorico è la sequenza $a$ e $v$ (se questa è la lettura corretta) incisa su una fuseruola rinvenuta in una sepoltura bolognese databile fra secondo quarto e metà del VII secolo a. C. $(R E E 80,1)$. L'isolamento del documento e l'incertezza nella lettura della sequenza rendono però aleatoria ogni ulteriore deduzione, anche perché l'alta cronologia sembrerebbe indirizzare a una fase forse anche anteriore alla decantazione del primo alfabeto teorico. 
mente, indizia contestuale espunzione di san): una scelta forte, non scontata, che suggerisce la nascita immediata di un alfabeto teorico stabile con forte valore identitario in senso contrastivo. L'aspetto certamente più caratteristico della scrittura sabina è una rifunzionalizzazione dei grafemi "morti" molto più spinta di qualunque altro fenomeno simile documentato in Italia, e più vicina alle esperienze dell'Asia minore: il $\langle\theta>$ diventa $<$ í $>$, e il $<\chi>$ diventa $<$ ú $>$. Se la prima transizione è facilitata dalla contiguità nella sequenza alfabetica (con effetto di trascinamento nella recitazione), la seconda è aiutata: (1) dalla possibile assimilazione visiva $\mathrm{di}\langle\chi\rangle$ (a tridente) con una $<\mathrm{u}\rangle$ marcata da un tratto diacritico, e: (2) da una probabile debolezza strutturale del valore fonico del grafema, facilitata dalla sua posizione in coda alla sequenza, e indiziata anche dal suo reimpiego in funzione vocalica in altre scritture di derivazione greca (lidio, in parte anche il licio, in concorrenza però con un grafema simile con valore $/ \mathrm{k} /$ ). Non inganni, in questo senso, la posizione dei due grafemi, in versione modernizzata, in coda alla sequenza osca: l'osco è un costrutto tardo e artificiale, che mescola volutamente modelli diversi, nel contesto di una prepotente affermazione identitaria che non disdegna soluzioni fortemente contrastive (grafema di $<\mathrm{d}>$ !). A quanto è dato sapere, le due lettere caratteristicamente sabine furono aggiunte all'alfabeto osco dopo la sua prima formazione, e per questo accodate alla sequenza preesistente. ${ }^{39}$

7. Da quello che si è visto, è chiaro che la formazione di qualunque scrittura comporta un livello più o meno elevato di agency da parte di chi riceve questo strumento, quanto meno al momento dell'elaborazione di un alfabeto teorico stabile. Le congruenze e le divergenze non sono mai casuali, ma portano un significato di affermazione identitaria, i cui sottintesi storico-culturali sono spesso evidenti, e talora anche positivamente documentati. Chi riceve la scrittura non è un allievo incondizionatamente prono all'autorità del maestro, ma è egli stesso un futuro maestro. Questo è evidente quando l'elaborazione del nuovo sistema scrittorio, nella sua conclamata individualità, è immediata; altre volte però il processo è più lungo, e passa per una prima fase nella quale il modello è ritenuto nella sua interezza, come corpus dottrinale: questo è il caso dell'etrusco, anche se i primi scribi etruschi non possono certo essere biasimati, considerato che l'alfabeto greco, quando arriva in Etruria, è esso stesso ancora in cerca della propria identità. Superato questo primo periodo, però, nel corso della prima metà del VII secolo nasce il primo alfabeto teorico

39 Su tutta la questione si rimanda a Benelli c. s. b. 
etrusco, che è opera di maestri che volutamente ritengono al suo interno una schiera di grafemi "muti" (teoricamente non più necessari una volta abbandonato lo stato fluido del corpus dottrinale). Cosa può avere portato alla sua creazione?

La risposta può essere una sola. Il primo alfabeto teorico etrusco, che nasce certamente nella fascia più meridionale dell'Etruria, precocemente molto attiva nella produzione epigrafica, nasce con precisi intenti di interoperabilità. Nelle intenzioni dei suoi creatori, evidentemente, lo stesso alfabeto doveva servire a notare non solo l'etrusco, ma anche il greco, ${ }^{40}$ il latino, il falisco e le lingue sabelliche. ${ }^{41}$ Non a caso la Cerveteri orientalizzante ha restituito almeno un'iscrizione greca ${ }^{42} \mathrm{e}$, probabilmente, anche una latina ${ }^{43}$ realizzate nella città stessa. Se coglie nel vero l'ipotesi prospettata supra ( $\$ 6.5 . \mathrm{d}$, “soluzione b"), forse nell'estremo nord del mondo etrusco fu creato un primo alfabeto teorico diverso, destinato a venire incontro ad esigenze diverse di interoperabilità, nei confronti di gruppi umani parlanti lingue diverse.

Solo con il passaggio al VI secolo il mondo cosmopolita delle aristocrazie orientalizzanti, erede di una poleogenesi che coinvolse certamente gruppi etnico-linguistici eterogenei, si fuse definitivamente in una nuova identità, ormai univocamente etrusca. Tuttavia, nella fascia più meridionale della regione, anche dopo che la "scuola veiente", intorno al 600 a.C., aveva ridotto l'alfabeto teorico ai soli grafemi provvisti di potenziali corrispondenze operative nella lingua etrusca, la prassi epigrafica cerite-veiente rimase significativamente caratterizzata da un rifiuto sistematico della distinzione grafica delle due si-

40 La persistente vicinanza fra la scrittura etrusca e quella greco-euboica si misura sul fatto che non mancano iscrizioni di interpretazione problematica. Un esempio è il graffito edito da Del Verme e Sacco 2005, 252, n. 1, interpretato come euboico (ibid., 258-259) per la sua provenienza (Cuma), ma in realtà certamente etrusco per le evidenti considerevoli difficoltà di lettura in greco già sottolineate in sede di edizione. Altro esempio è il graffito pure cumano ET $\mathrm{Cm} 6.3$, per il quale coesistono interpretazione greca (Cassio 1993-1994) ed etrusca (Colonna 1995).

41 Si veda a tal proposito la condizione ambigua dell'alfabetario di Capena CIE $8547=E T$ Fa 9.2, che viene a volte considerato come etrusco (come mostra, ad es., l'inclusione in $E T$ ), ma più spesso una sorta di costrutto ibrido, per la presenza di forme dichiaratamente sabelliche ( $h$ a rettangolo vuoto) e falische ( $f$ a freccia): $c f$. per esempio Gaucci 2012, 60; Maras 2013, 270.

42 La celebre firma del vasaio Aristonothos, su cui p. es. Cordano 2007.

43 Si tratta dell'iscrizione di Tita Vendia, per la quale si è anche proposta una lettura in falisco, anche se l'opzione latina è oggi preferita dai più (da ultimo Bakkum 2009, 583584, con bibliografia precedente). La provenienza da Cerveteri, tuttavia, pur essendo molto verosimile, non è certa. 
bilanti sorde (nonostante i grafemi necessari fossero tutti presenti in sede di alfabeto teorico) e da un parallelo rifiuto della piena accoglienza del grafema $<\mathrm{f}\rangle$, quasi a voler mantenere una piena riconoscibilità reciproca con sistemi di altre lingue. ${ }^{44}$ L'interoperabilità sembra quindi una delle preoccupazioni principali nella storia della scrittura etrusca, con una significativa persistenza del principio nella fascia più meridionale della regione molto più che altrove; solo il tardo arcaismo mise fine a questo stato di cose.

\section{$\begin{array}{llllllllllll}\mathbf{B} & \mathbf{I} & \mathbf{B} & \mathbf{L} & \mathbf{I} & \mathbf{O} & \mathbf{G} & \mathbf{R} & \mathbf{A} & \mathbf{F} & \mathbf{I} & \mathbf{A}\end{array}$}

Adiego 2018: I. X. Adiego, "Local adaptations of the alphabet among the non-Greek peoples of Anatolia”, in: S. Ferrara e M. Valério (eds.), Paths into script formation in the Ancient Mediterranean [Studi Micenei ed Egeo-Anatolici, n.s., Supplemento 1], Roma 2018, 145-162.

Attenni e Maras 2005: L. Attenni e D. F. Maras, "Materiali arcaici dalla collezione Dionigi di Lanuvio ed il più antico alfabetario latino", SE 70, 2004 [2005], 61-78.

Bagnasco Gianni 2008: G. Bagnasco Gianni, “Comunicare per immagini: una questione di alfabeto", Aristonothos 3, 2008, 47-72.

Bakkum 2009: G. C. L. M. Bakkum, The Latin dialect of the ager Faliscus. 150 years of scholarship, Amsterdam 2009.

Bartoloni 1997: G. Bartoloni, "La Tomba dell'Alfabeto di Monteriggioni”, in: Etrusca et Italica. Scritti in ricordo di Massimo Pallottino, Pisa-Roma 1997, 25-49.

Bellelli e Benelli 2018: V. Bellelli e E. Benelli, Etruschi. La scrittura, la lingua, la società, Roma 2018.

Benelli 2013: E. Benelli, “Lettera sul vaso di legno Kat. 106”, in: A. Babbi e U. Peltz (ed.), La Tomba del Guerriero di Tarquinia. Identità elitaria, concentrazione del potere e networks dinamici nellavanzato VIII sec. a.C. Das Kriegergrab von Tarquinia: Eliteidentität, Machtkonzentration und dynamische Netzwerke im späten 8. Jh. v. Chr., Mainz 2013, 86-87.

Benelli c. s. a: E. Benelli, "Scrittura e cultura epigrafica nell'Etruria orientalizzante. Appunti di metodo sulle origini della scrittura etrusca e sui primi passi dell'epigrafia", in: The Orientalizing cultures in the Mediterranean, $8^{\text {th }}-6^{\text {th }}$ cent. $B C$. Origins, cultural contacts and local developments: the case of Italy (Atti del Convegno, Roma 2017), in corso di stampa.

Benelli c. s. b: E. Benelli, "Formazione e diffusione delle scritture centro-italiche. Lo stato della documentazione", in: L'Italie préromaine et la France. Un regard français sur l'Italie préromaine: mélanges en l'honneur de Dominique Briquel, in corso di stampa.

44 Scopo raggiunto tanto efficacemente che è stato possibile interpretare complessivamente come etruschi tutti i graffiti arcaici da Roma (tranne uno) presentati in REE 79, 31-52, laddove molti di essi saranno più probabilmente latini. Ma le sigle monoletterali e $\mathrm{i}$ testi disperatamente frammentari restano ambigui, e solo in quattro casi su ventuno l'attribuzione all'etrusco può considerarsi certa. 
Benelli c. s. c: E. Benelli, "Scrittura degli scribi e scrittura delle città. Per una fenomenologia della scrittura nell'Italia preromana”, in: Schriftkonventionen in pragmatischer Perspektive. Akten der Arbeitstagung der Indogermanischen Gesellschaft, Brüssel 2018, in corso di stampa.

Cappuccini 2018: L. Cappuccini, "Un kyathos di bucchero da Poggio Pelliccia. La "bottega vetuloniese" e il suo ruolo nella trasmissione della scrittura in Etruria", SE 80, 2017 [2018], 61-82.

Cassio 1993-1994: A. C. Cassio, "La più antica iscrizione greca di Cuma e tív(v)vuat in Omero", Die Sprache 35, 1991-1993 [1993-1994], 187-207.

CIE: Corpus Inscriptionum Etruscarum.

Colonna 1995: G. Colonna, "Etruschi a Pitecusa nell'Orientalizzante Antico", in: A. Storchi Marino (ed.), L'incidenza dellantico. Studi in memoria di Ettore Lepore, Napoli 1995, 325342.

Colonna 2016: G. Colonna, "L'iscrizione di Osteria dell'Osa", in: G. Colonna, Italia ante Romanum imperium. Scritti di antichità etrusche, italiche e romane (1999-2013), 5-6, Pisa-Roma 2016, 987-991 [= G. Colonna, "Intervento", in: G. Bartoloni e F. Delpino (ed.), Oriente e Occidente: metodi e discipline a confronto. Riflessioni sulla cronologia dell'età del Ferro italiana. Atti dell'incontro di studi (Roma, 30-31.10.2003), Pisa-Roma 2005, 478-483].

Cordano 2007: F. Cordano, "Aristonothos. La firma", Aristonothos 1, 2007, I-III.

Cubberley 1996: P. Cubberley, “The Slavic Alphabets”, in: P. T. Daniels e W. Bright (eds.), The world's writing systems, New York-Oxford 1996., 346-355.

Daniels e Bright 1996: P. T. Daniels e W. Bright (eds.), The world's writing systems, New YorkOxford 1996.

D’Acunto 2013: M. D’Acunto, Il mondo del vaso Chigi. Pittura, guerra e società a Corinto alla metà del VII secolo a.C., Berlin-Boston 2013.

Del Verme e Sacco 2005: L. Del Verme e G. Sacco, "Cuma: frammenti ceramici iscritti dagli scavi dell'Orientale”, AIONArch 9-10, 2005, 251-270.

ET: G. Meiser (Hrsg.), Etruskische Texte, Hamburg $2014^{2}$.

Farrujia de la Rosa et alii 2010: A. J. Farrujia de la Rosa, W. Pichler, A. Rodrigue e S. García Marín, "The Libyco-Berber and Latino-Canarian scripts and the colonization of the Canary Islands", African Archaeologial Review 27, 2010, 13-41.

Franc 2018: E. Franc, “Appunti sull'ipotesi di un valore identitario dell'alfabeto di Lugano", QuadFriulA 28, 2018, 123-132.

Gaucci 2012: A. Gaucci, "Alfabetari latini nell'Italia preromana”, Atti e memorie della Accademia Petrarca di lettere, arti e scienze 72-73, 2012, 59-83.

Guarducci 1987: M. Guarducci, L'epigrafia greca dalle origini al tardo Impero, Roma 1987.

Guillaume-Pey 2018: C. Guillaume-Pey, "Des rébelles armés de feuilles blanches aux déchiffreurs de pierres. Appropriation de l'écriture chez les Sora et d'autres groupes tribaux de l'Inde (Adivasi)", Terrain 70, 2018, 62-81.

Holisky 1996: D. A. Holisky, “The Georgian Alphabet”, in: P. T. Daniels e W. Bright (eds.), The world's writing systems, New York-Oxford 1996, 364-370.

Janko 2017: R. Janko, "From Gabii and Gordion to Eretria and Methone: the Rise of the Greek Alphabet”, in: J. Strauss Clay, I. Malkin e Y. Z., Tzifopoulos (eds.), Panhellenes at Methone. Graphê in Late Geometric and Protoarchaic Methone, Macedonia (ca 700 BCE), BerlinBoston 2017, 135-164. 
Kelly 2018a: P. Kelly, “The invention, transmission and evolution of writing: insights from the new scripts of West Africa", in: S. Ferrara e M. Valério (eds.), Paths into script formation in the Ancient Mediterranean [Studi Micenei ed Egeo-Anatolici, n.s., supplemento 1], Roma 2018, 189-209.

Kelly 2018b: P. Kelly, “The art of not being legible: invented writing systems as technologies of resistance in South East Asia", Terrain 70, 2018, 38-61.

Kenzelmann-Pfyffer, Theurillat e Verdan 2005: A. Kenzelmann-Pfyffer, T., Theurillat e S., Verdan, "Graffiti d'époque géométrique provenant du sanctuaire d'Apollon Daphnéphoros à Erétrie”, ZPE 151, 2005, 51-83.

Langdon 2005: M. K. Langdon, “A new Greek abecedarium”, Kadmos 44, 2005, 175-182.

Maras 2013: D. F. Maras, “Questioni di identità: Etruschi e Falisci nell'agro falisco”, in: G. Cifani (ed.), Tra Roma e l'Etruria. Cultura, identità e territorio dei Falisci, Roma 2013, 265-285.

Maras 2014a: D. F. Maras, "Le iscrizioni”, in: L. M. Michetti e I. van Kampen (ed.), Il tumulo di Monte Aguzzo a Veio e la collezione Chigi. Ricostruzione del contesto dellolpe Chigi e note sulla formazione della collezione archeologica della famiglia Chigi a Formello, Roma 2014, 141-149.

Maras 2014b: D. F. Maras, “Breve storia della scrittura celtica d'Italia. L'area golasecchiana”, in: Zixu. Studi sulla cultura celtica di Golasecca, I, Roma 2014, 79-93.

Marinetti 2013: A. Marinetti, "Parole dal passato: tra insegnamento e pratica”, in: Venetkens. Viaggio nella terra dei Veneti antichi (Catalogo della Mostra, Padova 2013), Venezia 2013, 302-305.

Méndez Dosuna 2017: J. Méndez Dosuna, "Methone of Pieria: a Reassessment of Epighraphical Evidence (with a Special Attention to Pleonastic Sigma)", in: J. Strauss Clay, I. Malkin e Y. Z. Tzifopoulos (eds.), Panhellenes at Methone. Graphê in Late Geometric and Protoarchaic Methone, Macedonia (ca. 700 BCE), Berlin-Boston 2017, 242-260.

Menuta 2016: F. Menuta, "Over-differentiation in Amharic orthography and attitude towards reform”, Ethiopian journal for social sciences and language studies 3/1, 2016, 3-32.

Pandolfini e Prosdocimi 1990: M. Pandolfini e A. L. Prosdocimi, Alfabetari e insegnamento della scrittura in Etruria e nell'Italia antica, Firenze 1990.

Prosdocimi 1990: A. L. Prosdocimi, "Insegnamento e apprendimento della scrittura nell'Italia antica”, in: Pandolfini e Prosdocimi 1990, 155-298.

REE: "Rivista di Epigrafia Etrusca”, SE.

Sanjian 1996: A. K. Sanjian, “The Armenian Alphabet”, in: P. T. Daniels e W. Bright (eds.), The world's writing systems, New York-Oxford 1996, 356-363.

Sassatelli 1984: G. Sassatelli, "Graffiti alfabetici e contrassegni nel villanoviano bolognese. Nuovi dati sulla diffusione dellalfabeto in Etruria padana”, EmPrerom 9/10, 1981-82 [1984], 147-255.

Solinas 2015: P. Solinas, "Sull'alfabeto del celtico d'Italia”, in: D. Baglioni e O. Tribulato (a cura di), Contatti di lingue, contatti di scritture. Multilinguismo e multigrafismo dal Vicino Oriente Antico alla Cina contemporanea, Venezia 2015, 107-122.

Strauss Clay, Malkin e Tzifopoulos 2017: J. Strauss Clay, I. Malkin e Y. Z., Tzifopoulos (eds.), Panhellenes at Methone. Graphê in Late Geometric and Protoarchaic Methone, Macedonia (ca 700 BCE), Berlin-Boston 2017.

Tuck e Wallace 2018: A. Tuck e R. Wallace, "A third inscribed kyathos fragment from Poggio Civitate", RM 124, 2018, 301-309. 
Enrico Benelli

Van Schaik 2011: S. van Schaik, "A new look at the Tibetan invention of writing", in: Y. Imaeda, M. T. Kapstein e T. Takeuchi (eds.), New studies of the old Tibetan documents: philology, history and religion, Tokyo 2011, 45-96.

Wachter 1989: R. Wachter, "Zur Vorgeschichte des griechischen Alphabets", Kadmos 28, 1989, 19-78.

Wachter 2001: R. Wachter, Non-Attic Greek vase-inscriptions, Oxford 2001.

Woodard 2014: R. D. Woodard, The textualization of the Greek alphabet, Cambridge 2014. 